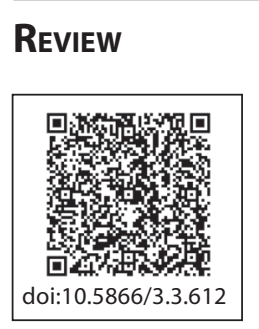

\title{
Radiation and Chemotherapy Complications and their possible management in head and neck region- an overview
}

\author{
Pankaj Agarwal' ${ }^{1}$, Juhi upadhyay ${ }^{2}$, Ashu Agarwal ${ }^{3}$
}

Department of Oral Pathology and Micro Biology KD Dental College, National Highway-II, Mathura, Uttara Pradesh - 282001

Reader $^{1}$

Asst. Professor ${ }^{2}$

Lecturer $^{3}$

\section{Article Info}

Received: April 15, 2011

Review Completed: May, 17, 2011

Accepted: June, 19, 2011

Available Online: October, 2011

(C) NAD, 2011 - All rights reserved

\begin{abstract}
:
Oral complications from radiation to the head and neck or chemotherapy for any malignancy can compromise patients' health and quality of life, and affect their ability to complete planned cancer treatment. For some patients, the complications can be so debilitating that they may tolerate only lower doses of therapy, postpone scheduled treatments, or discontinue treatment entirely. Oral complications can also lead to serious systemic infections. Medically necessary oral care before, during, and after cancer treatment can prevent or reduce the incidence and severity of oral complications, enhancing both patient survival and quality of life. In the present article we have tried to present an overview of oral complications and their possible management after radiation and chemotherapy in head and neck region.
\end{abstract}

Key words: Radiation therapy, chemotherapy, oral complications

\section{Introduction}

Head and neck radiation patients are a significant challenge relative to both intra therapy and post therapy oral complications resulting from radiation therapy. Unlike the oral complications of chemotherapy that are of shorter duration and are significant for only a short period (a few weeks to 2 months) after the cessation of therapy, the oral complications of head and neck radiation are more predictable, often more severe, and can lead to permanent tissue changes that put the patient at risk for serious chronic complications.

\section{Evaluation before the treatment}

Elimination of oral disease and implementation of oral care protocols designed to maintain maximum oral health must be a component of patient assessment and care prior to radiation therapy. During and after radiation therapy, oral management will be dictated by the specific needs of the patient, the specifics of the radiation therapy, and presence of chronic complications caused by

Email for correspondence:

drpankajagarwaldentist@yahoo.com radiation therapy. Ongoing oral assessment and treatment of complications are essential, because radiation to oral tissues typically renders patients at lifelong risk for oral complications. In addition, invasive oral procedures can cause additional sequelae. Dental care typically needs to be altered due to underlying chronic radiation-induced tissue damage.

Patients should receive a comprehensive oral evaluation several weeks prior to initiation of highdose upper-mantle radiation. This timing provides an appropriate interval for tissue healing in the event invasive oral procedures, including dental extractions, dental scaling/polishing, and endodontic therapy, are necessary. The goal of this evaluation is to identify teeth at significant risk for infection and/or breakdown that would ultimately require aggressive or invasive dental treatment during or after the radiation that increase the risk of soft tissue necroses and osteonecroses. The likelihood of these lesions occurring postradiation increases over the patient's lifetime as the risk of significant dental disease increases. This includes restorative, periodontal, and endodontic disease. Since xerostomia is an expected 
complication, it is especially important that preradiation dental care strategies to permanently reduce the impact of the complications of severe xerostomia and xerostomia decay are sought.

In addition, radiation-specific issues emerge:

1. Radiation injury is oral tissue-specific and is dependent on dosage and portals of therapy.

2. Duration of radiation-induced oral mucositis typically extends for 6 to 8 weeks, versus the approximate 5 to 14 days observed in chemotherapy patients. The extended radiation treatment protocols are chiefly responsible for this difference.

- The primary cause of oral cancer is tobacco use; alcohol abuse further escalates risk. It is therefore critical that the head/neck cancer patient permanently cease tobacco use.

- Most patients with smoking-related cancer appear motivated to quit smoking at the time of cancer diagnosis.

- Continued smoking substantially increases the likelihood of recurrence or occurrence of a second cancer in survivors, particularly in those who previously received radiation therapy.

- A stepped-care approach to tobacco cessation is recommended, including direct physician advice to quit and provision of basic information to all patients at each contact during the first month of diagnosis, followed by more intensive pharmacologic treatment or counseling for those having difficulty quitting or remaining abstinent.

\section{Oral Complications of Head and Neck Radiation}

The oral complications of head and neck radiation can be divided into two groups based on the usual time of their occurrence: acute complications occurring during therapy or late complications occurring after radiation therapy has ended.

Acute complications include oropharyngeal mucositis, sialadenitis and xerostomia, infections (primarily candidiasis), and taste dysfunction; occasionally tissue necrosis can be seen late during therapy, but this is relatively rare.
Chronic complications include mucosal fibrosis and atrophy, xerostomia, xerostomia caries, infections (primarily candidiasis), tissue necrosis (soft tissue necrosis and osteonecrosis), taste dysfunction (dysgeusia/ageusia), muscular and cutaneous fibrosis, ${ }^{1}$ and dysphagia. ${ }^{2}$

\section{Management of oral mucositis}

Mucositis, which can be caused by radiation or chemotherapy, results from the mitotic death of the basal cells of the mucosal epithelium. Portions of the gastrointestinal tract become inflamed, and red, burn-like or ulcer-like sores appear throughout the mouth (Figure 1.). The etiopathogenesis of head and neck radiation mucositis appears to be similar but not identical to mucositis caused by high-dose chemotherapy. ${ }^{3-6}$ Management strategies described for chemotherapy/hematopoietic stem cell transplantation are generally applicable to the head/ neck radiation patient. ${ }^{7-9}$ The extensive duration and severity of radiation mucositis combined with most radiation patients being treated as outpatients results in pain management challenges. As mucositis severity increases and topical pain management strategies become less effective, it becomes increasingly necessary to depend on systemic analgesics to manage oral radiation mucositis pain. ${ }^{10}$ Because there is generally no bleeding risk for head and neck radiation patients, analgesic treatment begins with nonsteroidal anti-inflammatory drugs (NSAIDs). As pain increases, NSAIDs are combined with opioids and patients can be made relatively comfortable. Doses for NSAIDs are titrated up to their recommended dosing ceiling; on the other hand, opioids are titrated to effective pain relief. Systemic analgesics are given by the clock to achieve steadystate blood levels to provide adequate pain relief. Additionally, adjunctive medications are given to provide adjuvant analgesia and manage side effects of NSAIDs and opioids. Zinc supplementation used with radiation therapy may improve mucositis and dermatitis. ${ }^{11}$ The use of alcohol-free povidone-iodine mouthwash can reduce the severity and delay the onset of oral mucositis due to antineoplastic radiation therapy. ${ }^{12}$

\section{Early infections}

Candidiasis is the most common clinical infection of the oropharynx in irradiated patients. 
Patients receiving head and neck radiation are frequently colonized with Candida, as demonstrated by an increase in quantitative counts and rates for clinical infection. ${ }^{13}$ Candidiasis may exacerbate the symptoms of oropharyngeal mucositis (Figure 2.).

Treatment of oral candidiasis in the radiation patient has primarily utilized topical antifungals such as nystatin and clotrimazole. Compliance can be compromised secondary to oral mucositis, nausea, pain, and difficulty in dissolving nystatin pastilles and clotrimazole troches. Use of systemic antifungals including ketoconazole and fluconazole to treat oral candidiasis has proved effective and may have advantages over topical agents for patients experiencing mucositis.

Bacterial infections may also occur early in the course of head/neck radiation and after appropriate diagnosis (i.e., culture and sensitivity tests) should be treated with antibiotics. Herpesvirus infections may also occur in patients who are seropositive prior to head and neck radiation. ${ }^{14,15}$

\section{Taste dysfunction}

As oral and pharyngeal mucosa are exposed to radiation, taste receptors become damaged and taste discrimination becomes increasingly compromised. ${ }^{16,17}$ After several weeks of radiation, it is common for patients to complain of no sense of taste. It will generally take upwards of 6 to 8 weeks after the end of radiation therapy for taste receptors to recover and become functional. Zinc sulfate supplements (220 mg 2 or 3 times a day) have been reported to help with recovery of the sense of taste. ${ }^{18-20}$

\section{Late reactions}

Late oral complications of radiation therapy are chiefly a result of chronic injury to vasculature, salivary glands, mucosa, connective tissue, and bone. ${ }^{21,22,18}$ Types and severity of these changes are directly related to radiation dosimetry, including total dose, fraction size, and duration of treatment. Mucosal changes include epithelial atrophy, reduced vascularization, and submucosal fibrosis. These changes lead to an atrophic, friable barrier. Fibrosis involving muscle, dermis, and the temporomandibular joint results in compromised oral function. Salivary tissue changes include loss of acinar cells, alteration in duct epithelium, fibrosis, and fatty degeneration. Compromised vascularization and remodeling capacity of bone leads to risk for osteonecrosis.

\section{Caries}

Dental-caries risk increases secondary to a number of factors including shifts to a cariogenic flora, reduced concentrations of salivary antimicrobial proteins, and loss of mineralizing components. ${ }^{18}$ Treatment strategies must be directed to each component of the caries process. Optimal oral hygiene must be maintained. Xerostomia should be managed whenever possible via salivary substitutes or replacements. Caries resistance can be enhanced with use of topical fluorides and/or remineralizing agents. Efficacy of topical products may be enhanced by increased contact time on the teeth by application using vinyl carriers. Patients not able to effectively comply with use of fluoride trays should be instructed to use brush-on gels and rinses.

Increased colonization with Streptococcus mutans and Lactobacillus species increases caries risk. Culture data can be useful in defining level of risk in relation to colonization patterns. Topical fluorides or chlorhexidine rinses may lead to reduced levels of $S$. mutans but not Lactobacilli. Due to adverse drug interactions, fluoride and chlorhexidine dosing should be separated by several hours.

Remineralizing agents, which are high in calcium phosphate and fluoride, have demonstrated salutary in vitro and clinical effects. The intervention may be enhanced by delivering the drug via customized vinyl carriers. This approach extends the contact time of active drug with tooth structure, which leads to increased uptake into enamel.

\section{Tissue necrosis}

Necrosis and secondary infection of previously irradiated tissue is a serious complication for patients who have undergone radiation for head and neck tumors. ${ }^{18}$ Acute effects typically involve oral mucosa. Chronic changes involving bone and mucosa are a result of the process of vascular inflammation and scarring that in turn result in hypovascular, hypocellular, and hypoxic changes. Infection secondary to tissue injury and osteonecrosis confounds the process (Figure 3).

Soft tissue necrosis can involve any mucosal surface in the mouth, though nonkeratinized surfaces 
appear to be at moderately higher risk. Trauma and injury is often associated with nonhealing soft tissue necrotic lesions, though spontaneous lesions are also reported. Soft tissue necrosis begins as an ulcerative break in the mucosal surface and can spread in diameter and depth. Pain will generally become more prominent as soft tissue necrosis becomes worse. Secondary infection is a risk.

As noted above, risk for necrosis is directly related to radiation dose and volume of tissue irradiated. The unilateral vascular supply to each half of the mandible results in osteonecrosis most frequently involving the mandible versus the maxilla.Presenting clinical features include pain, diminished or complete loss of sensation, fistula, and infection. Pathologic fracture can occur as the compromised bone is unable to appropriately undergo repair at the involved sites. Risk for tissue necrosis is in part related to trauma or oral infection; however, idiopathic cases can also occur. Patients who have received high-dose radiation to the head and neck are at risk for osteonecrosis for life, with an overall risk of approximately $15 \%$.

Ideally, osteonecrosis management centers on prevention that begins with comprehensive oral and dental care prior to radiation. The dentition, periodontium, periapices, and mucosa should be thoroughly examined to identify oral disease, which could lead to serious odontogenic, periodontal, or mucosal infections that could necessitate surgical therapy post radiation. Oral disease should be eliminated prior to cancer therapy. Dentition that exhibits poor prognosis and is within high-dose fields should be extracted prior to radiation therapy. Ideally, at least 7 to 14 days should be allowed for healing prior to initiation of radiation; some have suggested allowing up to 21 days. Surgical technique should be as atraumatic as possible and utilize primary wound closure.

Patients who develop osteonecrosis should be comprehensively managed to include elimination of trauma, avoidance of removable dental prosthesis if the denture-bearing area is within the osteonecrotic field, assurance of adequate nutritional intake, and discontinuation of tobacco and alcohol use. Topical antibiotics (e.g., tetracycline) or antiseptics (e.g., chlorhexidine) may contribute to wound resolution. Wherever possible, coverage of the exposed bone with mucosa should be achieved. Analgesics for pain control are often effective. Local resection of bone sequestrae may be possible.

Hyperbaric oxygen therapy (HBO) is recommended for management of osteonecrosis, although it has not been universally accepted. HBO has been reported to increase oxygenation of irradiated tissue, promote angiogenesis, and enhance osteoblast repopulation and fibroblast function. $\mathrm{HBO}$ is usually prescribed as 20 to 30 dives at $100 \%$ oxygen and 2 to 2.5 atmospheres of pressure. If surgery is needed, ten dives of postsurgical hyperbaric oxygen therapy are recommended. Unfortunately, HBO technology is not always accessible to patients who might otherwise benefit because of a lack of available units and the high price of care.

Partial mandibulectomy may be necessary in severe cases of osteonecrosis. The mandible can be reconstructed to provide continuity for esthetics and function. A multidisciplinary cancer team including oncologists, oncology nurses, maxillofacial prosthodontists, general dentists, hygienists, and physical therapists is appropriate for management of these patients.

\section{Dysfunction of the Jaws}

Musculoskeletal syndromes may develop secondary to radiation and surgery. Lesions include soft tissue fibrosis, surgically-induced mandibular discontinuity, and parafunctional habits associated with emotional stress caused by cancer and its treatment. Patients can be instructed in physical therapy interventions including mandibular stretching exercises as well as use of prosthetic aids designed to reduce severity of fibrosis. It is important that these approaches be instituted prior to trismus development. If clinically significant changes develop, several approaches including stabilization of occlusion, trigger point injection and other pain management strategies, muscle relaxants, and/or tricyclic medications can be considered.

\section{References}

1. Vera-Llonch $\mathrm{M}$, Oster $\mathrm{G}$, Hagiwara $\mathrm{M}$, et al.: Oral mucositis in patients undergoing radiation treatment for head and neck carcinoma. Cancer 2006; 106(2): 329-336.

2. Caudell JJ,Schaner PE,Meredith RF, et al.: Factors associated with long-term dysphagia after definitive radiotherapy for locally advanced head-and-neck cancer. Int J Radiat Oncol Biol Phys 2009; 73(2): 410-415. 
3. Sonis ST: Mucositis as a biological process: a new hypothesis for the development of chemotherapy-induced stomatotoxicity. Oral Oncol 1998; 34(1): 39-43.

4. Peterson DE: Research advances in oral mucositis.Curr Opin Oncol 1999; 1(4): 261-256.

5. Sonis ST, Peterson DE, McGuire DB, eds.: Mucosal injury in cancer patients: new strategies for research and treatment. J Natl Cancer Inst Monogr 2001; (29): 1-54.

6. Barasch A, Peterson DE: Risk factors for ulcerative oral mucositis in cancer patients: unanswered questions. Oral Oncol 2003; 39(2): 91-100.

7. Dose AM:The symptom experience of mucositis, stomatitis, and xerostomia. Semin Oncol Nurs 1995; 11 (4): 248-255.

8. Demarosi F, Bez C, Carrassi A: Prevention and treatment of chemo- and radiotherapy-induced oral mucositis. Minerva Stomatol 2002; 51(5): 173-186.

9. Shih A, Miaskowski C, Dodd MJ, et al.: A research review of the current treatments for radiation-induced oral mucositis in patients with head and neck cancer. Oncol Nurs Forum 2002; 29(7): 1063-1080.

10. Wong PC, Dodd MJ, Miaskowski C, et al.: Mucositis pain induced by radiation therapy: prevalence, severity, and use of self-care behaviors. J Pain Symptom Manage 2006; 32 (1): 27-37.

11. Lin LC, Que J, Lin LK, et al.:Zinc supplementation to improve mucositis and dermatitis in patients after radiotherapy for head-and-neck cancers: a double-blind, randomized study. Int J Radiat Oncol Biol Phys 2006; 65(3): 745-750.

12. Madan PD, Sequeira PS, Shenoy K, et al.:The effect of three mouthwashes on radiation-induced oral mucositis in patients with head and neck malignancies: a randomized control trial. J Cancer Res Ther 2008 Jan-Mar; 4(1): 3-8.

13. Epstein JB, Freilich MM, Le ND: Risk factors for oropharyngeal candidiasis in patients who receive radiation therapy for malignant conditions of the head and neck. Oral Surg Oral Med Oral Pathol 1993; 76(2): 169-174.

14. Oakley C, Epstein JB, Sherlock CH: Reactivation of oral herpes simplex virus: implications for clinical management of herpes simplex virus recurrence during radiotherapy. Oral Surg Oral Med Oral Pathol Oral Radiol Endod 1997; 84(3): 272-278.

15. Epstein JB, Gorsky M, Hancock $P$, et al.: The prevalence of herpes simplex virus shedding and infection in the oral cavity of seropositive patients undergoing head and neck radiation therapy. Oral Surg Oral Med Oral Pathol Oral Radiol Endod 2002; 94(6): 712-716.

16. Nelson GM: Biology of taste buds and the clinical problem of taste loss. Anat Rec 1998; 253(3): 70-78.

17. Zheng WK, Inokuchi A, Yamamoto T, et al.:Taste dysfunction in irradiated patients with head and neck cancer. Fukuoka Igaku Zasshi 2002; 93(4): 64-76.

18. Silverman S Jr: Complications of treatment. In: Silverman S Jr, ed.: Oral Cancer. 5th ed. Hamilton, Canada:BC Decker Inc, 2003, pp 113-128.
19. Ripamonti C, Zecca E, Brunelli $C$, et al.: A randomized, controlled clinical trial to evaluate the effects of zinc sulfate on cancer patients with taste alterations caused by head and neck irradiation. Cancer 1998; 82 (10): 1938-1945.

20. Silverman JE, Weber CW, Silverman S Jr, et al.: Zinc supplementation and taste in head and neck cancer patients undergoing radiation therapy. J Oral Med 1983 Jan-Mar; 38(1): 14-16,

21. Myers RA, Marx RE: Use of hyperbaric oxygen in postradiation head and neck surgery. $\mathrm{NCl}$ Monogr 1990; (9): 151-157.

22. Epstein JB, Wong $F L$, Stevenson-Moore $P$ : Osteoradionecrosis: clinical experience and a proposal for classification. J Oral Maxillofac Surg 1987; 45 (2): 104-110.

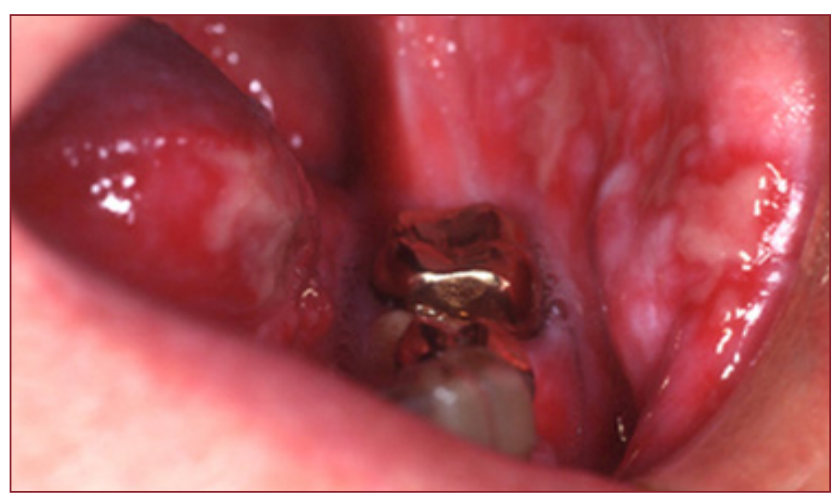

Figure 1. Oral mucositis involving buccal mucosa following radiation therapy.

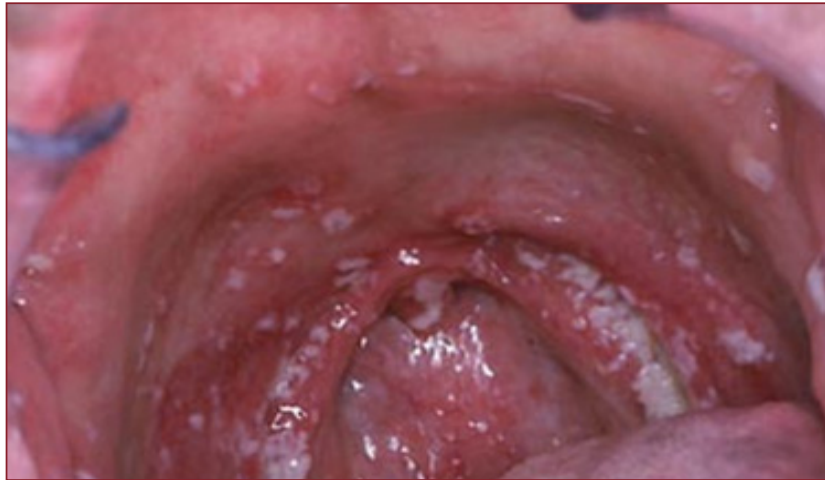

Figure 2. Candida infection following therapy.

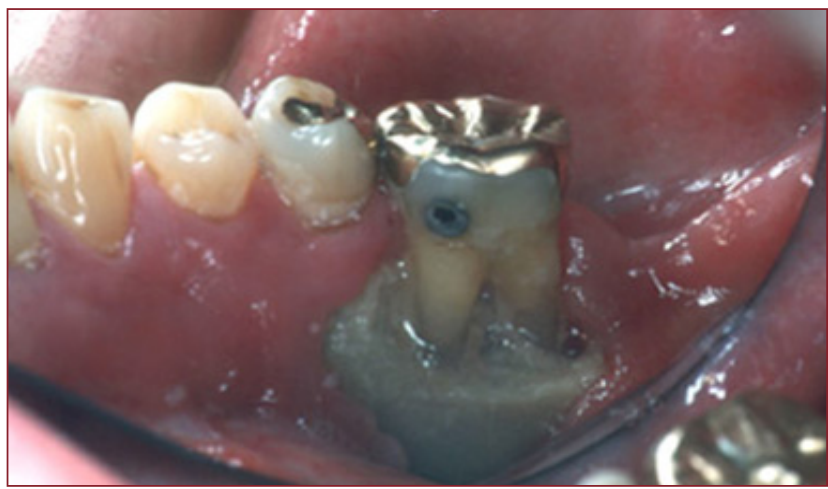

Figure 3. Osteoradionecrosis involving the mandible. 